\title{
Detection of Nicotine Effect on Colon Cells in a Plasmonic Platform
}

\author{
Tannaz Asadishad ${ }^{1}$, Foozieh Sohrabi ${ }^{1}$, Mohammad Hossein Ghazimoradi ${ }^{2,3}$, Seyedeh Mehri Hamidi ${ }^{*}$, Saeed \\ Javadi Anaghizi ${ }^{4}$, Shirin Farivar ${ }^{2,3}$
}

\author{
${ }^{1}$ Magneto-Plasmonic Lab, Laser and Plasma Research Institute, Shahid Beheshti University, Tehran, Iran \\ ${ }^{2}$ Laser Application in Medical Sciences Research Center, Shahid Beheshti University of Medical Sciences, Tehran, Iran \\ ${ }^{3}$ Life Science and Biotechnology Faculty, Shahid Beheshti University, Tehran, Iran \\ ${ }^{4}$ Central Laboratory of Shahid Beheshti University, Tehran, Iran
}

\section{*Correspondence to Seyedeh Mehri Hamidi, m_hamidi@sbu.ac.ir}

Published online January 18 2020

\begin{abstract}
Introduction: Smoking as one of the causes of various diseases has encouraged worldwide studies on its adverse pharmacological effects on different organs. Nicotine may influence the smooth muscles of the colon and subsequently the gut motility, which leads to a change in the moving rate of digested material through the gastrointestinal tract.

Methods: Among various techniques, optical detection methods benefit from non-contact and highsensitivity for studying the early effect of nicotine on the cells. Thus, we used an optically ellipsometric method to get the fast and sensitive nicotine effect on the colon cell. Two-dimensional plasmonic platforms by gold deposition onto the polydimethylsiloxane polymer (PDMS) patterned substrate were used as the guest medium of the cell and the sample was excited by all of the visible region wavelengths at different exposure time and maintenance time.

Results: Our results showed that the phase difference between each polarization increased by augmenting the exposure time of smoke over the cell at a fixed maintenance time and there was a general red-shift by increasing the maintenance time at a fixed exposure time.

Conclusion: Using different exposure time to cigarette smoke, we optically showed that the cigarette containing the addicting chemical of nicotine had a direct effect on the cultured colon cells on our 2D biocompatible plasmonic chip. It demonstrated considerable changes in the amplitude and phase of the interacted light by injecting nicotine into the system with the aid of the label-free and non-invasive plasmonic technique.
\end{abstract}

Keywords: Nicotine; Sensing; Surface plasmon polariton (SPP); Ellipsometry; Colon cell.

\section{Introduction}

As a stimulant and potent parasympathomimetic alkaloid, nicotine is made in the nightshade family of plants. As a smoking cessation aid, it can be used for the treatment of tobacco usage disorders. Nicotine is a basic part of tobacco smoke. ${ }^{1}$ This chemical plays the role of a receptor agonist at most nicotine acetylcholine receptors (nAChRs) ${ }^{2}$ except at 2 nicotinic receptor subunits (nAChRa9 and nAChRa10) where it performs as a receptor antagonist; therefore, nicotine is both a sedative and a stimulant based on the taken dose of nicotine and the individual's nervous system arousal. When a body is exposed to nicotine, the individual has a "kick" experience, which is partially due to adrenaline release caused by a nicotine stimulus. This surge of adrenaline stimulates the body and subsequently, such pharmacological effects as the prompt release of glucose and the subsequent increase in the heart rate, breathing activity, and blood pressure arise. In addition, nicotine causes the pancreas to produce less insulin, leading to a slight increase in blood sugar or glucose. Indirectly, nicotine provokes the release of dopamine in the pleasure and motivation areas of the brain, and similar to heroin and cocaine, it produces a pleasurable sensation. Nicotine is also believed to affect the smooth muscles of the colon and change the gut motility. Afterwards, the moving rate of food and digested material through the gastrointestinal tract alters. Smoking cigarettes, or any other tobacco products containing nicotine, release nitric oxide at various sites in the gastrointestinal tract. Nitric oxide causes the relaxation of the smooth muscles of the gastrointestinal tract and the reduction of muscular tone and activity. Recently there are more pieces of evidence showing that there is a direct relationship between smoking and different types of cancers like liver, colon, and stomach. ${ }^{3}$ There are various pieces of evidence presenting that nicotine not only increases tumor growth, but also plays a role in tumorigenesis. In addition, nicotine stimulates cells and promotes cancer cells ${ }^{4}$ and other types 
of cancer growth. As it is clear, the period of smoking is positively associated with the risk of colon stimulation. ${ }^{1}$

There are different ways to detect nicotine, including the nicotine blood test, the urine nicotine test, the saliva nicotine test, and the hair test. Although there are various strong existing methods of sensing and detection, they suffer from inadequate sensitivity, timeconsuming detection, the labeling process, high cost and so on. Optical spectroscopic methods have the virtue of being fast, non- invasive, and inexpensive. ${ }^{5,6}$ Using optical methods, we can reach higher potential for the real-time detection of nicotine effect on living cells in an easy and sensitive way. There are many methods for optical detection, including fluorescence detection, chemiluminescence and bioluminescence detection, Raman spectroscopy, and surface plasmon resonance. ${ }^{7}$ Among them, surface plasmon resonance is a proper candidate since it is a non-invasive, label-free and realtime technique for detecting the interaction between cells on the surface interface. ${ }^{8,9}$ There are various methods such as spontaneous reasoning, electroporation, or surface functionalization of the micro/nanostructures, which provide the possibility of investigating the intracellular medium. Plasmonic functionalization does not distribute the spontaneous electrical activity and it lets successive recording even during the reasoning process and can distinguish extracellular and intracellular contributions by means of partial cellular reasoning. Therefore, this method offers some worthwhile advantages compared to other methods like the continuous recording of electrical activity without disturbing it. ${ }^{10}$

In this study, we suggest the application of the plasmonic sensor to detect the changes in the live cultured colon cells due to exposure to cigarette smoke. We have proposed a method for quick detection of smoke effects on the cultured colon cells. In other words, this method helped us to examine the conditions when smoke was in contact with the cells. Using a two-dimensional (2D) soft plasmonic sensor and a plasmonic-ellipsometry technique, the sample was exposed to different flow rates of cigarette smoke containing the addicting chemical of nicotine. Considerable optical variation under different rates of nicotine provides us with information about the noticeable influence of nicotine on the colon cell in a new optical platform.

\section{Materials and Methods}

Fabrication of 2D Plasmonic Sensor and Cell Culture Benefiting from low cost, high throughput, and high resolution, nanoimprint lithography as a simple nanolithography technique was used to fabricate nanometer scale patterns. It yields patterns by the mechanical deformation of imprint resist which is generally a monomer or polymer curable by heat or UV light. Adhesion between the resist and the template is controlled by probable surface treatment to allow proper release. The direct imprint patterning method provides a monolithic integration alternative with better throughput. ${ }^{11,12}$

In this study, we benefited from a mold extracted from commercial camera CCDs with 2D patterns. Polydimethylsiloxane (PDMS) and its curing agent with the aspect ratio of 10:1 were mixed and poured on the mold. After degassing, the mold containing PDMS on its top was put on the heater at an average temperature of $80^{\circ} \mathrm{C}$ for about 1 hour. After cooling down, the patterned PDMS layer was removed from the mold following $30 \mathrm{~nm}$ gold sputtering. Afterwards, the chip was ready for the culturing of colon cells. The schematic of the fabrication process flow is shown in Figure 1A.

We purchase the DLD1 cell line from ATCC; this line is a human colorectal adenocarcinoma. The cells were cultured in DMEM containing 10\% FBS and 1\% Pen strep. After reaching a 90 percent confluence, we trypsinized and centrifuged them with $1000 \mathrm{rpm}$. Afterwards, we added a fresh medium and cultured them in the ratio of 1:5. For seeding the cells on chips, the chips were sterilized by UV and $75 \%$ ethanol. The chips were transferred into a culture plate and immobilized. After trypsinizing and centrifuge, we aspirated the medium, added the fresh medium, and resuspended the cells. The cells were transferred directly to the chips with an excess medium to cover the chips. For the validation of cell existence, SEM imaging was carried out. The SEM images of the chips with the cultured cells on top of it after being exposed to cigarette smoke are shown in Figure 1B.

For capturing smoke, we designed a chamber with four closed sides and one front-open side to embed the chip. There are two holes machined on the top face of the chamber; one hole for injecting the cell medium and the other for cigarette smoke entrance. The gold side of the chip faced the inner part of the chamber to have direct contact with the cell medium and smoke and the optical response collected from the backside of the sample.

\section{Experimental Setup and Measurement}

Ellipsometry is an optical technique for exploring the dielectric properties of the target sample by recording its reflection under $\mathrm{p}$ - and s- polarized incident light. ${ }^{13}$ Ellipsometry supplies valuable non-invasive amplitude and phase information from which one can determine the effective dielectric functions and their relationships to the material nanostructure and in our case, the plasmonic characteristics of the system. In general, this technique can be applied to characterize composition, roughness, thickness (depth), crystalline nature, doping concentration, electrical conductivity, and other material properties. Ellipsometry has also attracted the researchers' attention in other fields such as biology and medicine. ${ }^{14}$ The measured signal is the change in polarization as the incident radiation interacts with the target material. This polarization change is demonstrated by the amplitude 

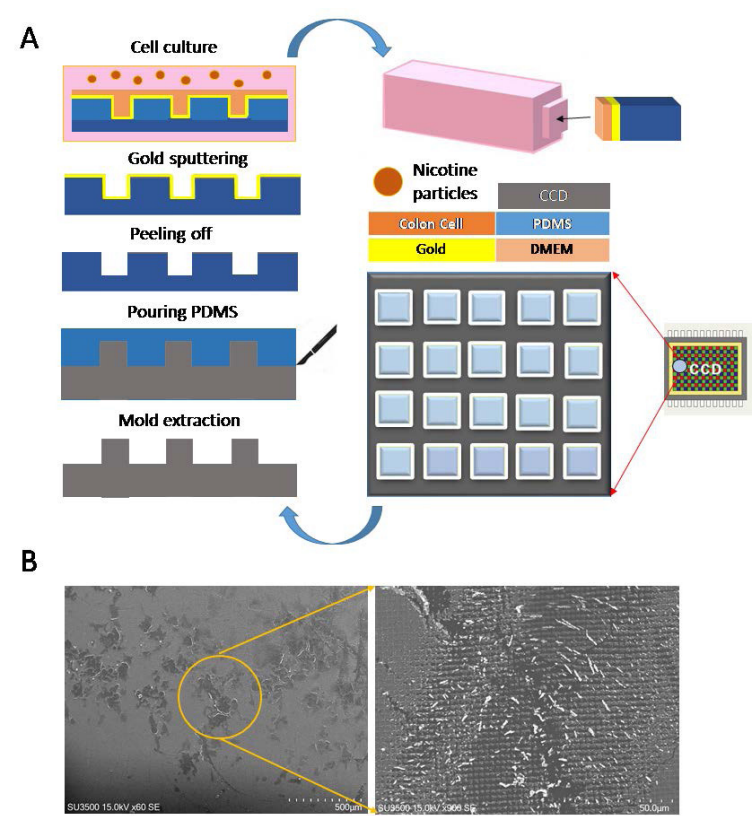

Figure 1. (A) the schematic diagram of the process flow for fabricating the $2 \mathrm{D}$ plasmonic chip which contains the subsequent processes of mold extraction from commercial CCD, PDMS pouring, degassing, curing, peeling off the cured PDMS layer, gold sputtering on the top surface of the patterned PDMS chip, and culturing of colon cells. Finally, the fabricated chip was inserted into the designed and machined chamber. (B) SEM images of the chips with cultured cells on top of it after being exposed to cigarette smoke.

ratio, $\Psi$, and the phase difference, $\Delta$. Since the signal depends on the thickness and the material properties, ellipsometry can be a universal tool for contact-free determination of optical properties of the target sample. Ellipsometry gives the complex reflectance ratio $\rho$ of a system parametrized by the amplitude component $\Psi$ and the phase difference $\Delta \cdot{ }^{15}$ The polarization state of the incident light upon the sample is decomposed into $s$ and $p$ components. The $s$ component is oscillating perpendicular to the plane of incidence and parallel to the sample surface, whereas the $p$ component is oscillating parallel to the plane of incidence. The amplitudes of the $s$ and $p$ components which are reflected and normalized to their initial value are denoted by $r_{s}$ and $r_{p}$ respectively. The incident angle is selected close to the Brewster angle of the sample to ensure a maximal difference between $r_{s}$ and $r_{p}^{13}$ :

$\rho=\frac{r_{p}}{r_{s}} \tan \Psi e^{i \Delta}$

The recorded reflection from the backside of the chip under $\mathrm{p}$ - and s-polarized incident light strongly depended on the characteristics of the adsorbed mass of biomolecules on the gold surface. ${ }^{16,17}$

A complex reflection coefficient, $\rho$, can be obtained using Fresnel coefficients as follows:

$$
\tan \psi=\rho=\frac{r_{p}}{r_{s}} \text { or } \frac{R_{p}}{R_{s}}
$$

Using $R_{s, p}=\left|r_{s, p} . r_{s, p}^{*}\right|$, we have:

$$
\begin{aligned}
& \left|r_{s, p}\right|=\sqrt{R_{s, p}} \rightarrow \tan \Psi=\frac{\sqrt{R_{p}}}{\sqrt{R_{s}}} \rightarrow \\
& \psi=\tan ^{-1} \sqrt{\frac{R_{p}}{R_{s}}}, \Psi \in\langle 0, \pi\rangle
\end{aligned}
$$

Where $r_{s, p}$ is Fresnel's coefficient. By applying the dependence on incident light frequency, we have:

$$
\begin{aligned}
& R_{s, p}(\omega)=\left|r_{s, p}(\omega) r_{s, p}^{*}(\omega)\right| \\
& r_{s, p}(\omega)=\left|r_{s, p}(\omega)\right| e^{i \theta_{s, p}(\omega)} \\
& r_{s, p}(\omega)=\sqrt{R_{s, p}} e^{i \theta_{s, p}(\omega)}
\end{aligned}
$$

By taking natural logarithm,

$$
\begin{aligned}
& \operatorname{Ln}\left[r_{s, p}(\omega)\right]=\operatorname{Ln}\left[\sqrt{R_{s, p}}\right]+i \theta_{s, p}(\omega) \\
& \theta_{s, p}(\omega)=-\frac{2 \pi}{\omega} P \int_{0}^{\infty} \frac{\operatorname{Ln}\left[\sqrt{R_{s, p}\left(\omega^{\prime}\right)}\right]}{\omega^{2}-\omega^{2}} d \omega^{\prime}+\theta_{0} .
\end{aligned}
$$

$\Delta$, as the phase difference between the $\mathrm{S}$ and $\mathrm{P}$ polarizations, equals

$$
\begin{aligned}
& \Delta=\theta_{p}(\omega)-\theta_{s}(\omega) \\
& =-\frac{2 \pi}{\omega} P \int_{0}^{\infty}\left[\frac{\operatorname{Ln}\left[\sqrt{R_{p}\left(\omega^{\prime}\right)}\right]}{\omega^{2}-\omega^{2}}-\frac{\operatorname{Ln}\left[\sqrt{R_{s}\left(\omega^{\prime}\right)}\right]}{\omega^{2}-\omega^{2}}\right] d \omega^{\prime}= \\
& -\frac{2 \pi}{\omega} P \int_{0}^{\infty}\left[\frac{\operatorname{Ln}\left[\sqrt{\left.\frac{R_{p}\left(\omega^{\prime}\right)}{R_{s}\left(\omega^{\prime}\right)}\right]}\right.}{\omega^{2}-\omega^{2}} .\right.
\end{aligned}
$$


An optical setup was mounted in order to record the reflection of the sample at an incident angle of $35^{\circ}$ with s- and p-polarized incident light. This optical setup consisted of a broadband halogen fiber optic illuminator, collimator, lens, Glan-Taylor calcite polarizer (GT10-A), aperture, sample chamber, rotation stage, Avantes spectrometer, cell medium injector, and smoke injector/ withdrawer. We designed an injection/suction pump for pulling cigarette smoke into/out of the chamber. For measurement, we kept the gas knob open for 1s and then closed it. Afterwards, we recorded the reflection spectrum at 1,60 , and 120 seconds after the closure of the smoke flow. At the second step, the gas knob was opened and kept in open status for 3 seconds, then closed and let spectrum recording after $1 \mathrm{~s}, 60 \mathrm{~s}$ and $120 \mathrm{~s}$. The same procedure was repeated for the open status of 5 seconds. The schematic diagram of the optical setup mounted for recording the reflection of the sensing chip under various exposure types and maintenance time of cigarette smoke is shown in Figure 2. In our FORTRAN written code, equations 1-5 were used to extract $\Delta$ and $\Psi$ parameters with respect to the incident wavelength.

\section{Results and Discussion}

We designed an injection/suction pump for pulling cigarette smoke into/out of the chamber. For measurement, we kept the gas knob open for $1 \mathrm{~s}$ and then closed it. Afterwards, we recorded the reflection spectrum at $1 \mathrm{~s}$, 60 s and 120s after the closure of the smoke flow. At the second step, the gas knob was opened and kept in open status for $3 \mathrm{~s}$, then closed and let spectrum recording after $1 \mathrm{~s}, 60 \mathrm{~s}$ and $120 \mathrm{~s}$. The same procedure was repeated for the open status of $5 \mathrm{~s}$. Therefore, the exposure time was $1 \mathrm{~s}, 3 \mathrm{~s}$ and $5 \mathrm{~s}$ and the maintenance time was $1 \mathrm{~s}, 60 \mathrm{~s}$ and 120s. Before cell culture, the reflection of the sensing chip was recorded with the injection of smoke with various exposure and maintenance time. The ellipsometric parameters were extracted using the recorded reflections under the impingement of $\mathrm{p}$ - and s-polarized incident light. The plasmonic resonance appeared as a dip in the reflection and $\Psi$ parameters since $\Psi$ value demonstrated the amplitude ratio of $\mathrm{p}$ - and s-polarized reflections. On the other hand, this dip in the $\Psi$ parameter is close to the peak value in the $\Delta$ parameter. As shown in Figure 3 , there are small changes in the value of $\Delta$ and $\Psi$

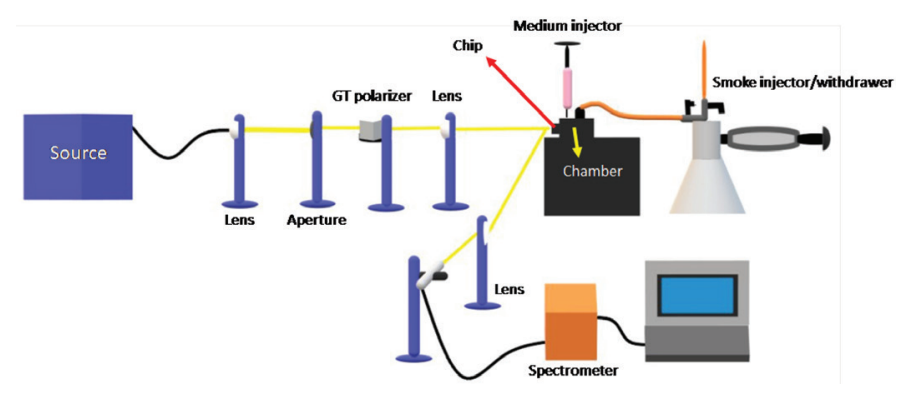

Figure 2. The Schematic Diagram of the Optical Setup Mounted for Recording the Reflection of the Sensing Chip Under Various Exposure Types and Maintenance Time of Cigarette Smoke.
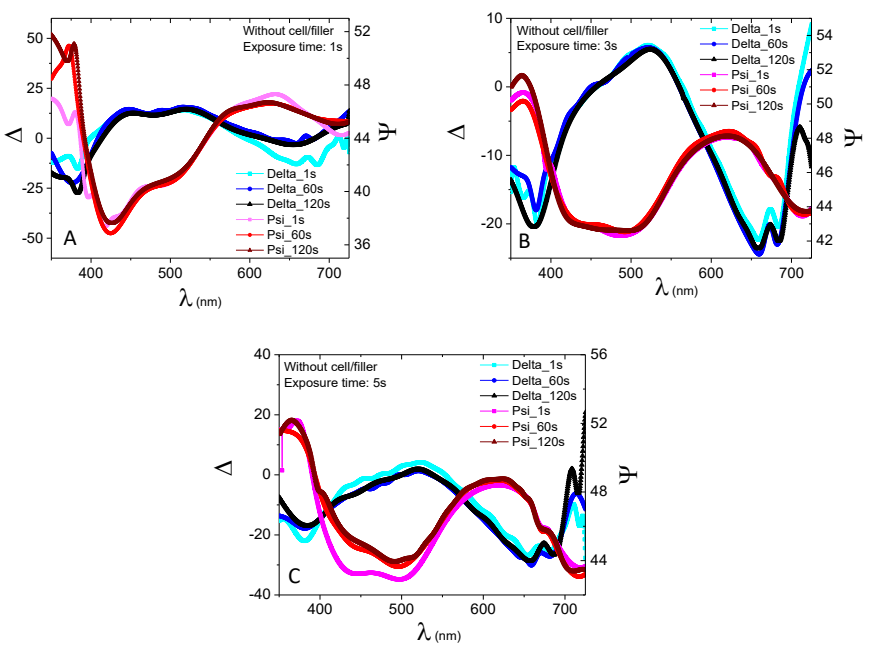

Figure 3. Extracted $\Delta$ Parameter of the Chip in the Presence of Cigarette Smoke With Maintenance Time of $1 \mathrm{~s}, 60 \mathrm{~s}$ and $120 \mathrm{~s}$ and Exposure Time of (A) $1 \mathrm{~s}$ (B) $3 \mathrm{~s}$ and (C) $5 \mathrm{~s}$. As observed, there are small changes in this parameter by increasing the exposure and maintenance time. However, there is a bit more variation in the $\Delta$ value when the exposure time was increased to $5 \mathrm{~s}$ with maintenance time of $1 \mathrm{~s}, 60 \mathrm{~s}$ and $120 \mathrm{~s}$. 

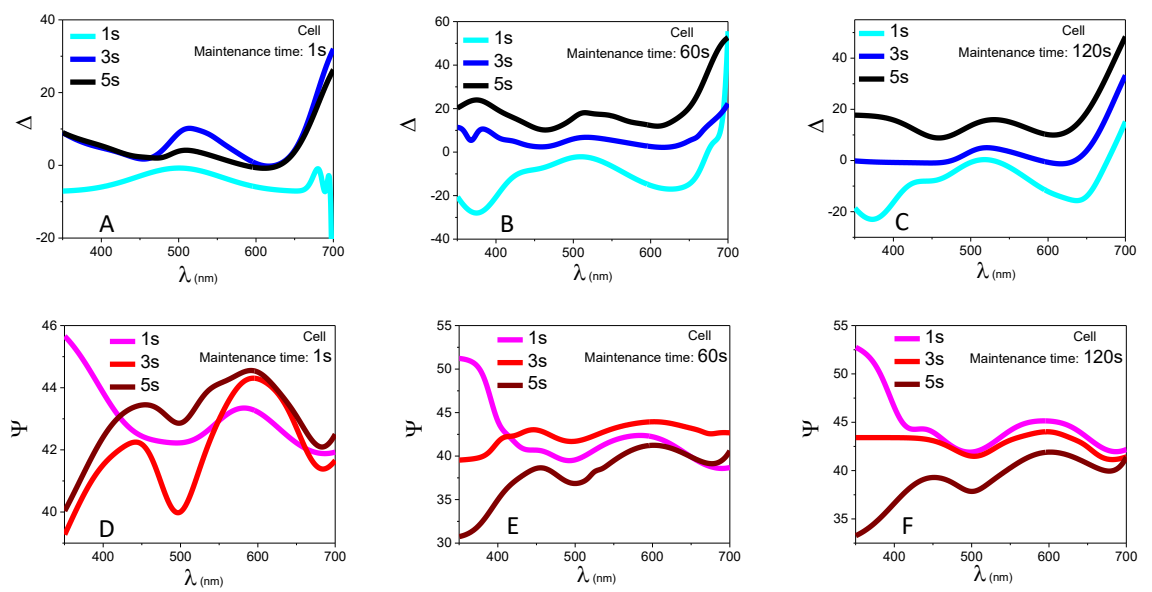

Figure 4. Extracted $\Delta(\mathrm{A}-\mathrm{C})$ and $\Psi$ (D-F) Parameters of the Chip in the Presence of Cigarette Smoke With the Exposure Time of $1 \mathrm{~s}, 3 \mathrm{~s}$ and $5 \mathrm{~s}$ and the Maintenance Time of (A, D) $1 \mathrm{~s}$ (B, E) $60 \mathrm{~s}$ and (C, F) $120 \mathrm{~s}$.
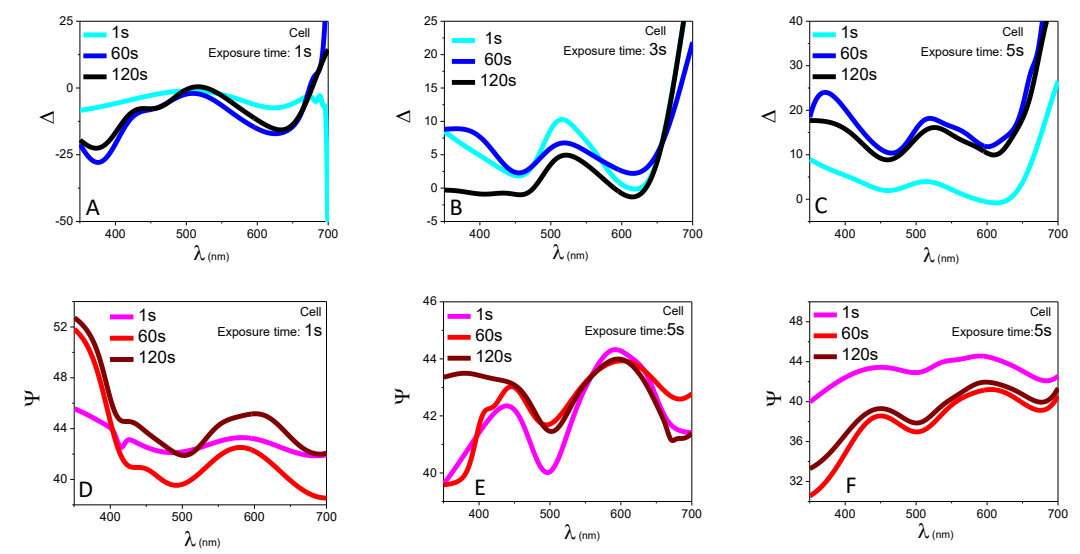

Figure 5. Extracted $\Delta(\mathrm{A}-\mathrm{C})$ and $\Psi$ (D-F) Parameters of the Chip in the Presence of Cigarette Smoke With Maintenance Time of $1 \mathrm{~s}, 60 \mathrm{~s}$ and $120 \mathrm{~s}$ and Exposure Time of (A, D) 1s (B, E) 3 and (C, F) $5 \mathrm{~s}$.

parameters under different exposure and maintenance smoke time. However, there is a bit more variation in the $\Delta$ value when the exposure time was increased to $5 \mathrm{~s}$ with the maintenance time of $1 \mathrm{~s}, 60 \mathrm{~s}$ and $120 \mathrm{~s}$.

However, in the case with cultured colon cells on the chips, there are noticeable splitting in $\Delta$ and $\Psi$ values under different exposure and maintenance time besides the shifts in the plasmonic resonance wavelength around $500 \mathrm{~nm}$ (Figures 4 and 5). For investigating the effect of increasing the exposure and maintenance time, the shift in the resonance wavelength location of $\Delta(\mathrm{A})$ and $\Psi$ (B) parameters is shown in Figure 6. As observed, there is a general red-shift in plasmonic resonance by increasing maintenance and exposure time. In other words, at a fixed maintenance time, by increasing the exposure time, the graph is vertically lifted up. In addition, at a fixed exposure time, by increasing the maintenance time, there is a general red-shift. This means that the maximum applied exposure time lets more smoke enter the chamber and interact with the cells. On the other hand, the maximum maintenance time provided sufficient reaction time for the cells. So, both these investigations show that smoke concentration and interaction duration affect noticeably the colon cells in an adverse manner.

\section{Conclusion}

Plasmonics is one of the highly-sensitive techniques that can be applied in a label-free and non-invasive platform to record the changes in biological samples. In this study, we used a combinatory method of plasmonic-ellipsometry to investigate the effect of cigarette smoke on DLD1 cells. Under different exposure and maintenance time of cigarette smoke, we observed considerable changes in extracted ellipsometric parameters of $\Delta$ and $\Psi$. By increasing the maintenance and exposure time, a general red-shift in plasmonic resonance was observed. Therefore, $\Delta$ and $\Psi$ increased by augmenting the exposure time at a fixed maintenance time. On the other hand, there was a general red-shift by increasing the maintenance time at a fixed exposure time. Maximum exposure time 

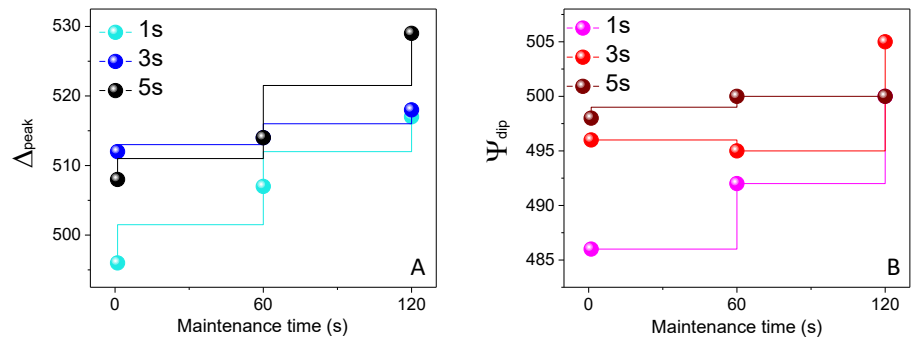

Figure 6. Step Graph of the Resonance Wavelength (A) $\Delta_{\text {peak }}$ and (B) $\Psi_{\text {dip }}$ With Respect to Increasing Exposure (1 s, $3 \mathrm{~s}$ and $5 \mathrm{~s}$ ) and Maintenance Time (1 s, 60 $\mathrm{s}$ and $120 \mathrm{~s}$ )

means more smoke entrance into the chamber and more interaction with the cells. In addition, the maximum maintenance time provided sufficient reaction time for the cells. This major change could be used for investigating the cells in different physiological conditions caused by cigarette smoke with various intensity and exposure time. In brief, we showed that the smoke concentration and interaction duration with alive colon cells considerably affect this biological sample. We hope this study can open new avenues toward rapid diagnosis under various physiological conditions and treatment efficiencies.

\section{Ethical Considerations}

This study has been approved by the ethical committee of Shahid Beheshti University.

\section{Conflict of Interests}

The authors declare no conflict of interest.

\section{Acknowledgement}

This work was supported by Iran National Science Foundation (INSF) under the grant number of 97006157.

\section{References}

1. Wong HP, Yu LE, Lam EK, Tai EK, Wu WK, Cho $\mathrm{CH}$. Nicotine promotes colon tumor growth and angiogenesis through $\beta$-adrenergic activation. Toxicol Sci. 2007;97(2):279-87. doi: 10.1093/toxsci/kfm060

2. Wong HP, Yu L, Lam EK, Tai EK, Wu WK, Cho CH. Nicotine promotes cell proliferation via $\alpha 7$-nicotinic acetylcholine receptor and catecholamine-synthesizing enzymesmediated pathway in human colon adenocarcinoma HT29 cells. Toxicol Appl Pharmacol. 2007;221(3):261-7. doi: 10.1016/j.taap.2007.04.002

3. Yi NY, Liu ES, Shin VY, Wu WK, Luo JC, Cho CH. Nicotine promoted colon cancer growth via epidermal growth factor receptor, c-Src, and 5-lipoxygenase-mediated signal pathway. J Pharmacol Exp Ther. 2004;308(1):66-72. doi: 10.1124/jpet.103.058321

4. Dasgupta P, Rizwani W, Pillai S, Kinkade R, Kovacs M, Rastogi $\mathrm{S}$, et al. Nicotine induces cell proliferation, invasion and epithelial-mesenchymal transition in a variety of human cancer cell lines. Int J Cancer. 2009;124(1):36-45. doi: $10.1002 /$ ijc. 23894

5. Stockman MI. Nanoplasmonic sensing and detection. Science. 2015;348(6232):287-8. doi: 10.1126/science. aaa6805

6. Van Der Pol E, Hoekstra AG, Sturk A, Otto C, Van Leeuwen TG, Nieuwland R. Optical and non-optical methods for detection and characterization of microparticles and exosomes. J Thromb Haemost. 2010;8(12):2596-607. doi: 10.1111/j.1538-7836.2010.04074.x

7. Sohrabi F, Hamidi SM, Mohammadi E. Role of higher order plasmonic modes in one-dimensional nanogratings. Opt Quant Electron. 2019;51(7):241. doi: 10.1007/s11082019-1958-x

8. Dan-Qun H, Zhen L, Hou CJ, Jun Y, Xiao-Gang L, HuanBao F, et al. Recent advances on optical detection methods and techniques for cell-based microfluidic systems. Chinese J Anal Chem. 2010;38(9):1357-65. doi: 10.1016/S18722040(09)60067-0

9. Sohrabi F, Hamidi SM. Fabrication methods of plasmonic and magnetoplasmonic crystals: a review. Eur Phys J Plus. 2017;132(1):15. doi: 10.1140/epjp/i2017-11294-2

10. Dipalo M, Amin H, Lovato L, Moia F, Caprettini V, Messina $\mathrm{GC}$, et al. Intracellular and extracellular recording of spontaneous action potentials in mammalian neurons and cardiac cells with 3D plasmonic nanoelectrodes. Nano lett. 2017;17(6):3932-9. doi: 10.1021/acs.nanolett.7b01523

11. Kaestner M, Krivoshapkina Y, Rangelow IW. Chapter 14, Next generation lithography-the rise of unconventional methods? In: Frontiers of Nanoscience. Vol. 11. Elsevier; 2016. p. 479-495. doi: 10.1016/B978-0-08-100354-1.000144.

12. Zou Y, Moreel L, Lin H, Zhou J, Li L, Danto S, et al. Solution Processing and Resist-Free Nanoimprint Fabrication of Thin Film Chalcogenide Glass Devices: InorganicOrganic Hybrid Photonic Integration. Adv Opt Mater. 2014;2(8):759-64. doi: 10.1002/adom.201400068

13. Sohrabi F, Hamidi SM. Optical detection of brain activity using plasmonic ellipsometry technique. Sensor Actuat B-Chem. 2017;251:153-63. doi: 10.1016/j.snb.2017.05.037

14. Tompkins H, Irene EA. Handbook of Ellipsometry. William Andrew; 2005.

15. Sohrabi F, Hamidi SM, Asgari N, Ansari MA, Gachiloo R. One dimensional photonic crystal as an efficient tool for in-vivo optical sensing of neural activity. Opt Mat. 2019; 96:109275. doi: 10.1016/j.optmat.2019.109275

16. Sohrabi F, Hamidi SM. Neuroplasmonics: from Kretschmann configuration to plasmonic crystals. Eur Phys J Plus. 2016;131(7):221. doi: 10.1140/epjp/i2016-16221-5

17. Walsh GF, Forestiere C, Dal Negro L. Plasmon-enhanced depolarization of reflected light from arrays of nanoparticle dimers. Opt Express. 2011;19(21):21081-90. doi: 10.1364/ OE.19.02108 